\title{
Ageing matrix promotes metastasis
}

\section{6 \\ changes in the \\ skin micro- \\ environment \\ associated \\ with ageing \\ promote \\ melanoma \\ metastasis}

Older patients with melanoma have lower disease-specific survival than younger patients, yet our understanding of how ageing affects tumour progression is limited. Two studies from Ashani Weeraratna's group have examined how changes in the skin microenvironment associated with ageing promote melanoma metastasis and modify immune infiltration.

Kaur et al. examined extracellular matrix (ECM) changes that might affect melanoma metastasis. Analyses of secreted proteins from human fibroblasts isolated from young ( $<45$ years) and aged ( $>55$ years) healthy donors indicated significant differences in many proteins involved in ECM remodelling. Young and aged fibroblasts also organized their ECM differently: the cell-derived matrix (CDM) of young fibroblasts was crosslinked and dense, whereas aged fibroblasts produced a more aligned CDM. These differences in ECM architecture were also confirmed in mouse skin.

Hyaluronan and proteoglycan link protein 1 (HAPLN1) was the most significantly decreased protein in the aged fibroblast secretome. Addition of recombinant HAPLN1

(rHAPLN1) to aged fibroblasts in vitro or to the skin of older mice reduced the CDM alignment and increased crosslinking, mimicking younger CDM.

Melanoma cells were more invasive in in vitro skin reconstructions built with aged fibroblasts, so the authors hypothesized that HAPLN1 loss might promote cell invasion. Indeed, melanoma cells plated on aged fibroblast-derived CDM were less invasive if rHAPLN1 was added during ECM production. In addition, tumours formed from YUMM1.7 mouse melanoma cells in aged mice were smaller, and the mice had fewer metastases in the lungs if the mice were treated with rHAPLN1 prior to YUMM1.7 cell injection.

rHAPLN1 did not affect cell proliferation or apoptosis, suggesting that the effects on primary tumour growth were a result of changes to the microenvironment, possibly to immune cell infiltration. $\mathrm{T}$ cell motility towards melanoma cells was reduced in the presence of aged fibroblast media in tumour spheroid and skin reconstruction assays, and this was reversed by addition of rHAPLN1. Increased $\mathrm{CD}^{+}$and $\mathrm{CD} 8^{+}$ $\mathrm{T}$ cell infiltration into YUMM1.7 tumours was also observed in aged mice treated with rHAPLN1.

The authors propose that the opposite effects of HAPLN1 on cancer cell and $\mathrm{T}$ cell migration relate to the inability of cancer cell nuclei to pass through a dense, crosslinked ECM.

Ecker et al. investigated the observation that older patients with melanoma have fewer metastases in the sentinel lymph node (LN), yet have a higher incidence of distal metastases and a worse disease-specific survival compared with younger patients. These data were recapitulated in the YUMM1.7 mouse model; aged mice had fewer LN metastases but more lung metastases.

The authors hypothesized that increased lymphatic permeability might explain these observations, and noted that LNs from older patients have decreased ECM complexity compared with those from younger patients with melanoma. In vitro analysis confirmed that endothelial permeability is increased in the presence of ECM from aged fibroblasts. Following on from the observations of Kaur et al., they found that loss of HAPLN1 increased endothelial permeability and the ability of melanoma cells to cross this barrier. This increase in permeability was also linked to reduced vascular endothelial (VE)-cadherin-dependent cellcell adhesion and reduced cell-ECM adhesion through $\alpha 1$ and $\beta 1$ integrins.

HAPLN1 expression was reduced in LNs from aged mice and humans with melanoma, and direct analysis of patient LNs confirmed that those with lower HAPLN1 expression had increased lymphatic permeability and worse overall survival than those with higher HAPLN1 expression. In aged mice, LN injection of rHAPLN1 increased the number of LN metastases but reduced metastasis to the lung. These data support a model of sequential melanoma metastasis first to LNs and then to distant sites.

Overall, these studies highlight the importance of ECM architecture in melanoma progression and provide a mechanistic explanation for how ECM breakdown that occurs with ageing might increase distant metastasis.

Sarah Seton-Rogers

ORIGINAL ARTICLES Ecker, B. L. et al. Agerelated changes in HAPLN1 increase lymphatic permeability and affect routes of melanoma metastasis. Cancer Discov. https://doi.org/ 10.1158/2159-8290.CD-18-0168 (2018) | Kaur, A. et al. Remodeling of the collagen matrix in aging skin promotes melanoma metastasis and affects immune cell motility. Cancer Discov. https://doi.org/10.1158/2159-8290.CD-18-0193 (2018) 